\title{
Design and analysis of dual band pass filter
}

\author{
Amina Puran ${ }^{1 *}$, Şehabeddin Taha İmeci ${ }^{2}$ \\ ${ }^{1,2}$ Department of Electrical and Electronics Engineering, International University of Sarajevo, Bosnia
}

*Corresponding author: aminapuran@gmail.com
Abstract
2020.
Published by
ARDA.
In this work, a dual-band meandered line bandpass filter is designed and
includes mostly geometry and some material characteristics like dielectric
thickness and metal types. This filter has a compact structure which is one of the
main benefits. It has many applications in the industry and that is what makes
work on it very interesting. Values for the S11 parameter are $-20.70 \mathrm{~dB}$ and -41.72
dB for $4.9 \mathrm{GHz}$ and $5.5 \mathrm{GHz}$, respectively. Values for S12 parameter are $-0.03 \mathrm{~dB}$
and $-2.91 \mathrm{e}-4 \mathrm{~dB}$ for $4.9 \mathrm{GHz}$ and $5.5 \mathrm{GHz}$, respectively.

Keywords: Filter, Dual bandpass filter, Microstrip, Cut-off frequency, Q factor; microwave; Multi-band operation

\section{Introduction}

Dual bandpass filter is a simple design of microstrip filter. It is very compact with box dimensions $40 \mathrm{x} 40 \mathrm{~mm}$. Main applications are reflected in manufacturing wireless transmitters and receivers [1]. Real circuit of dual band pass filter represents a combination of the low-pass filter and high-pass filter, which means that at certain range of frequency we will have two reversed signals. In practice ideal band-pass filter does not exist so we expect certain loss in output signal [2]. Frequency ranges are given in $\mathrm{dB}$ which is the most convenient way for our design and analysis. Since this is dual band-pass filter, we have two cut-off frequencies. Important characteristics of every band pass filter is Q factor. Our design has low Q factor which means that it gives narrow band pass frequency range. This important factor gives us possibility to name our design narrow band pass filter. Main characteristics of design are given as follows:

- Dielectric constant $\varepsilon_{r}=4.4$ (Quartz-fused)

- Frequency range of $4.8-4.9 \mathrm{GHz}$ and $5.4-5.5 \mathrm{GHz}$

- Box size 40x40mm

- Cell size $0.2 \times 0.2 \mathrm{~mm}$

\section{Design methodology}

Design is shown in Fig. 1 while output response is shown in Fig. 2. Design and analysis are done in Sonnet software [3]. Since nowadays communication circuit weights a lot, many dual band circuits are created to reduce its volume, including antennas, couplers and filters [4]. Band pass filter represent main component in filtering unwanted frequencies in those systems. In practice of dual band pass filters, strong attenuation within two passbands is required. The whole design must be perfectly symmetric as seen in Fig. 1. Comparison of

This work is licensed under a Creative Commons Attribution License (https://creativecommons.org/licenses/by/4.0/ ) that allows others to share and adapt the material for any purpose (even commercially), in any medium with an acknowledgement of the work's authorship and initial publication in this journal. 
this dual band pass filter with conventional ones shows that dual band pass filter is smaller in size. Due to the high demand for compact high-performance in wireless communication systems, dual-mode microstrip filters have been widely studied by numerous researchers for its advantages in applications requiring filter with features such as small size, low mass and low loss [5]. Nevertheless, while building this filter, we must predict some variations which can appear due to parasitic coupling on input and output.

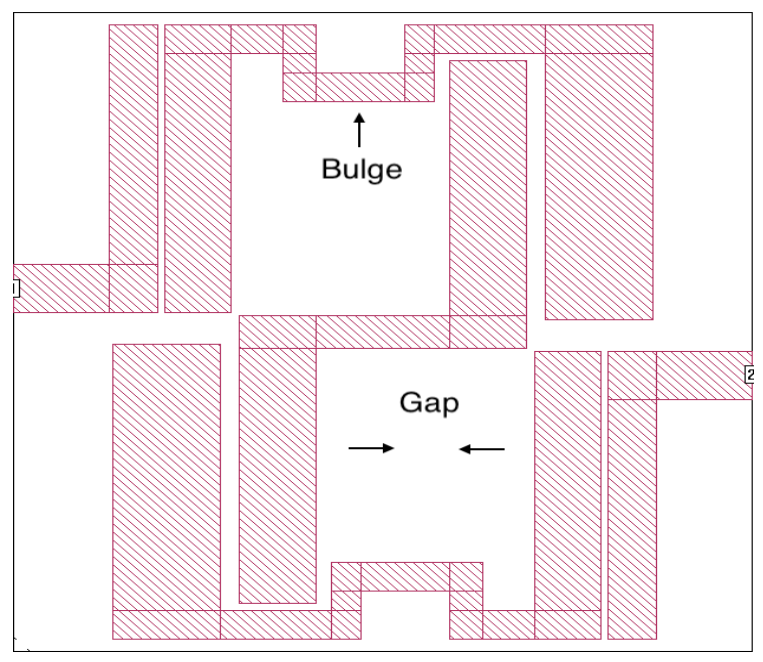

Figure 1. Design of the dual band pass filter

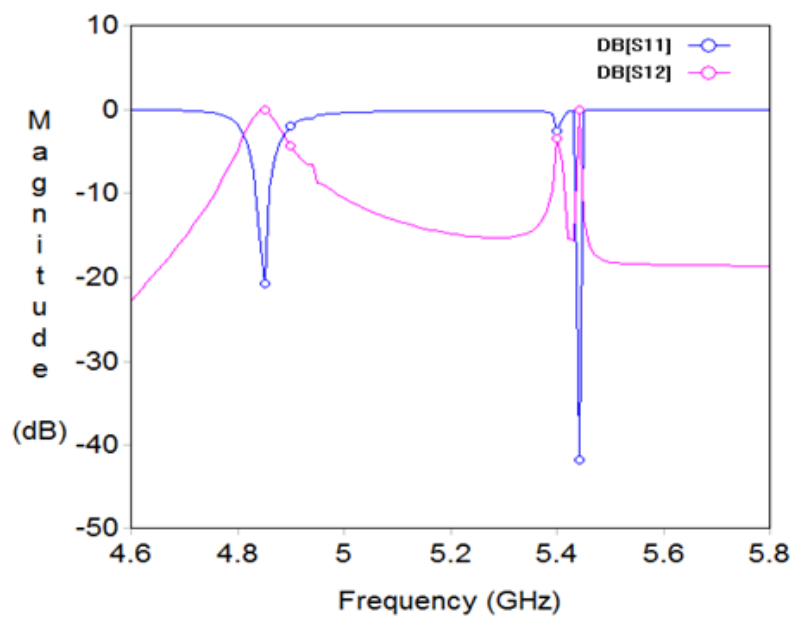

Figure 2. Output signal of the dual band pass filter simulation

There are several techniques to design RF filters and those are Butterworth filter, Chebyhev filter, Linear Phase filter and Ellipctic filter [6]. Our design does not follow any of them specifically, but we made design on our own while adjusting some of the parameters. Its dual property can be developed using several methods as cascading or connecting in the parallel two dissimilar BPFs with common input/output coupling structure [7]. As measure unit millimeters are chosen since those are the most convenient according to European standards and our proposed design. For dielectric material Fr-4 with dielectric constant 4.4 is chosen since it is commonly used, easiest for fabrication and gives good performance. Similar situation is with choosing the metal type of the filter. Copper is found to be the best since it is cheapest and gives good performance. Source impedance is given as usual common value of $50 \mathrm{Ohms}$. For cell size, $0.2 \mathrm{~mm}$ is taken. It gives fast response of simulation since our box size is quite small. If you choose larger, simulations take much more time to finish, making filter slower and unstable with huge deviations in output signal. Elements from which electronic filters can be made are resistors, capacitors, inductors, transistors and operational amplifiers [8]. 
However, this filter can be designed from low pass filter with changing equivalent circuit. Replace an inductor with series LC circuit and replacing a capacitor with parallel LC circuit of corresponding values [9]. As defined this kind of the filter is used to suppress specific range of frequency but at same time to pass other ranges. From its definition we can conclude that it is noisier than another simple band pass or band stop signal, especially when we have narrow band.

\section{Parameters variations}

As mentioned above main studies are done examining the changes in geometry. Dual band pass filters are very demanding in industry since they provide functionality of two separate filters in the size of the one [10]. The main parameters to evaluate the performance of any filter are:

- input match (expressed as ratio of reflected power and power transmitted to the source)

- insertion loss (comparison between power transferred to the load and load received power)

- VSWR (Voltage Standing Wave Ratio)

The S parameters (input match and insertion loss) are important concept in microwave design since it is easy to measure and indicate the results of output signal. Note that values for $\mathrm{S}$ parameters are given in decibels (dB). Input match represents the amount of power reflected back to the source, while insertion loss is amount of the power lost due to the components combination while transferred to the load. VSWR is comparison between the maximum voltage amplitude towards standing wave minimum voltage amplitude [11].

We expect small value for the input match for better performance. Since we express values in decibels, we aim to the negative values for input match. Insertion loss represents the power lost in a process of transfer a power due to its components. Output of the signal is shown in Fig. 2 in the form of Cartesian plot. Blue line represents S11 (input match) and pink S12 (insertion loss or attenuation of the signal). VSWR has a maximum when reflected wave and incident wave are in phase, while minimum will occur if those waves are in opposite phases. While analyzing we notice that changes in thickness affect output signal.

The larger thickness produces better output signal. However, since the changes in thickness affect other physical parameters, we must make trade-off between filter's geometry and find output signal with best properties. By searching the parts of the design which affect the largest changes we found that bulge and the gap are the best ones to examine as seen in Fig. 1. Further, we have done analysis by changing dielectric thickness. Table 1, 2 and 3 provide information about signals while changing distance of the bulge, gap and dielectric thickness respectively. Increasing the bulge distance resulted in very unstable results for S11 parameter in the $2^{\text {nd }}$ frequency range. Although, changes in other parameters are quite predictable.

Table 1. Changing distance of the bulge

\begin{tabular}{lllll}
\hline Distance & S11 & S12 & S11 & S12 \\
\hline $0.3 \mathrm{~mm}$ & -20.70 & -0.03 & -41.72 & $-2.9 \mathrm{e}-4$ \\
$0.32 \mathrm{~mm}$ & -22.63 & -0.02 & -21.37 & $-1.3 \mathrm{e}-4$ \\
$0.34 \mathrm{~mm}$ & -22.44 & -0.02 & -17.39 & -0.07 \\
$0.36 \mathrm{~mm}$ & -20.19 & -0.04 & -19.63 & -0.04 \\
$0.38 \mathrm{~mm}$ & -17.84 & -0.07 & -14.28 & -0.16 \\
\hline
\end{tabular}

As mentioned above, even small changes in geometry affect final output signal. Filters are sensitive devices. From all geometry changes we have done; largest changes are in variety of bulge and gap (see Fig. 1). We were decreasing the gap width and results are shown in Table 2. 
Table 2. Changing the gap width

\begin{tabular}{lllll}
\hline Gap & S11 & S12 & S11 & S12 \\
\hline $11.8 \mathrm{~mm}$ & -20.70 & -0.03 & -41.72 & $-2.9 \mathrm{e}-4$ \\
$12.0 \mathrm{~mm}$ & -20.07 & -0.04 & -16.19 & -0.10 \\
$12.2 \mathrm{~mm}$ & -30.20 & $-4.1 \mathrm{e}-3$ & -2.93 & -3.09 \\
$12.4 \mathrm{~mm}$ & -33.50 & $-1.9 \mathrm{e}-3$ & -2.12 & -4.12 \\
$12.6 \mathrm{~mm}$ & -27.09 & $-8.4 \mathrm{e}-3$ & -9.4 & -26.72 \\
\hline
\end{tabular}

Referent dielectric thickness is 1.60 . We made very small changes in increasing and decreasing that value by $0.1 \mathrm{~mm}$. Results are shown in Table 3.

Table 3. Changing dielectric thickness

\begin{tabular}{lllll}
\hline Dielectric T. & S11 & S12 & S11 & S12 \\
\hline & & & & \\
$1.58 \mathrm{~mm}$ & -35.85 & $-1.1 \mathrm{e}-3$ & -18.40 & -0.06 \\
$1.59 \mathrm{~mm}$ & -28.37 & $-4.2 \mathrm{e}-3$ & -12.03 & -16.39 \\
$1.60 \mathrm{~mm}$ & -20.70 & -0.03 & -41.72 & $-2.9 \mathrm{e}-4$ \\
$1.61 \mathrm{~mm}$ & -18.09 & -0.06 & -10.03 & -16.21 \\
$1.62 \mathrm{~mm}$ & -23.07 & -0.01 & -16.75 & -1.02 \\
\hline
\end{tabular}

Although obtained results provide good output signal, real results may differ from it due to the dielectric loss, edge capacitance, etc. [11].

\section{Numerical analysis and equivalent circuit}

Dual band pass filters are developed using multi-resonant circuit technique. This technique includes several steps as follows:

- design the individual passband filter (combining lowpass filter with bandpass filter and those transformations)

- combine these two filters [5].

Fig. 3 shown is equivalent circuit of the dual band pass filter. It is taken by cascading multiple band pass filters. Band pass filter allows certain band of frequency to pass while at same time blocks remaining range of frequency. It results in larger size because there is a use of diplexer type structures in order to match impedance of two filters. Among new versions of band pass filters introduced is open stub cascades structures which produce more complex filter with larger size. Similar to the band pass filter there is band stop filter, which aims to block certain range of frequency and pass remaining. However, to design dual band pass filter which represents the combination of two band pass filters, needed is to combine two equivalent circuits of band pass filters. Those will pass two bands at two different frequency ranges. To achieve two passbands simultaneously two resonators of the same layer are needed [4]. In wireless systems dual band pass filters are located at the beginning of those systems, enabling selection of frequency range at which certain system operates. Since there are numerous methods to produce dual band pass filter, we will discuss advantages and limitations of each method. Direct cascading of the two pass band filters will produce good dual results but filter will be huge in size which is not demanding in the modern technologies. Next method is by direct cascading of a bandstop filters. This type will also produce dual property with more compact size. Except these, there are methods like combining stop band filter with shunt stubs of unequal length [12]. Recently, 
new method is developed and it includes specification of central frequency and bandwidth to developed filter with dual property. These types are developed mostly because of its compact small size. Filters with closed loop resonator and coupled line are two polarized. Since we want to achieve high $\mathrm{Q}$ factor, ring resonator is used, and to satisfy dual property double ring resonator is connected in series with coupled lines. Besides, this kind of constructions results in small radiation losses. Manipulating the center frequency produces dual band property in its response. Currently, filters with easily controlled resonant frequencies are popular. They are constructed using stub loaded resonators, stepped impedance resonators and crossed resonators. In some filters, changing the length of stub loaded resonators can manipulate pass band frequency. This property makes dual band filters with stepped impedance and stub loaded resonators very attractive in the market. However, two bandwidths of this type of the filters cannot be controlled independently, which is also case with filter we proposed. This occurs due to two coupling paths. When resonator is symmetrical for further analysis can be used even and odd analyzing techniques. Dual band pass filters are composed of passive components which are able to separate frequency ranges differentiating wanted and unwanted signals. Further, they have to be accurate in order to produce good overall performance of the system. When it comes to open loop rectangular resonators as basic structure for constructing band pass filters, they show resonant characteristics which explain that transmission factor has maximal value at certain frequency and depends on its ports. Microstrip structure position of the resonant frequency depends on the size and length of the loop. However, it is not the only characteristic it depends on. Big role plays thickness and permittivity of the substrate. They affect the overall size of the filter. If we have two open loop resonators near each other, they will interact. Because of that fact, coupling between those two loops is very important. Nevertheless, whenever we combine two filters there is a need for proper isolation. In design of the band pass filter the area of the filter and performance are the two most important factors.

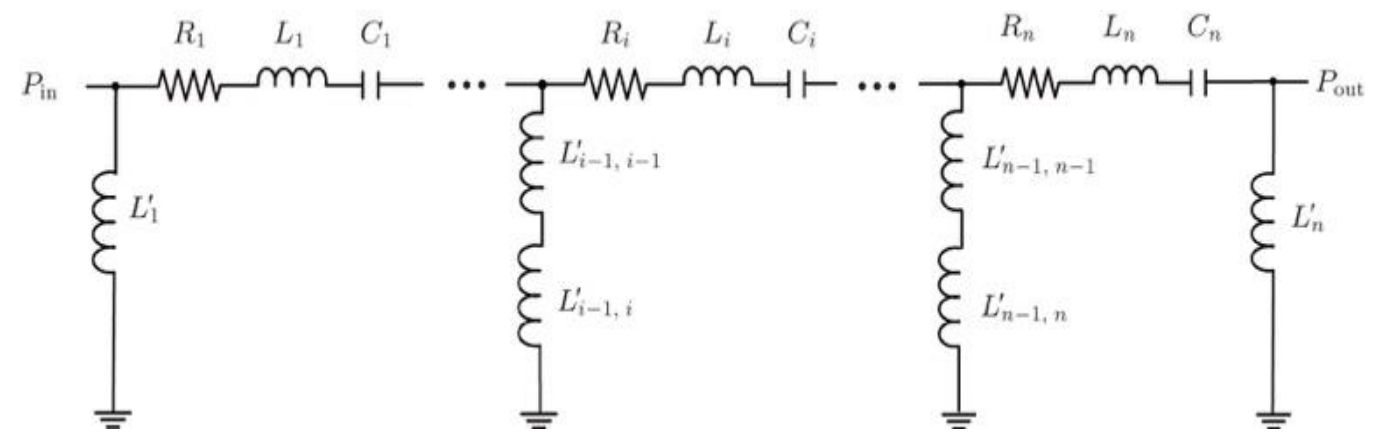

Figure 3. General equivalent circuit of the dual band pass filter [13]

To the given equivalent circuit, we represent numerical model for achieving values of its components. Equations 1, 2, 3 and 4 show relations used to achieve values for $L_{s}, C_{s}, L_{p}$ and $C_{p}$, respectively.

$$
\begin{gathered}
L_{s}=\frac{K_{i} L_{n}}{2 \pi B} \\
C_{S}=\frac{B}{2 \pi F_{u} F_{l} L_{n} K_{i}} \\
L_{p}=\frac{B K_{i}}{2 \pi B K_{i}} \\
C_{p}=\frac{C_{n}}{2 \pi B K_{i}}
\end{gathered}
$$

In equations above, $B$ stands for bandwidth while $K_{i}$ is denormalization constant $K_{i}=\frac{1}{Z_{o}}$. As mentioned previously, to analyze certain filter it is important to determine the quality factor. Equations 5 and 6 are used to determine coupling coefficients while equation 7 stands for external quality factor $Q$. 


$$
\begin{gathered}
M_{1,1}=M_{2,2 \prime}=M_{3,3 \prime}=J \sqrt[4]{\frac{L_{1} L_{a}}{C{ }_{1} C_{a}}} \\
M_{1,2}=M_{2,3}=J_{12}^{\prime} \sqrt{\frac{L_{1}^{\prime}}{C_{1}}}(6) \\
Q_{e x}=\frac{\omega_{0} C_{1}^{\prime}}{J_{01}^{\prime}}
\end{gathered}
$$

$J$ inverter is required in order to achieve a dual band pass filter with coupled resonator. It is achieved by transforming mixed series with parallel dual resonator circuit in two resonators of the same type coupled through a $J$ inverter [14]. There are two ways to achieve this type of the filter, either with cross-coupling or using simple folded hald-wavelength resonators. To decrease the size of the filter, coupling structures ends are curled among each other. Those ends must be connected through cascaded structures to fulfil different impedance characteristics. When it comes to irregularity of the thickness of those two filters are solved with including the equations 8 and 9 for even and odd mode, respectively.

$$
\begin{aligned}
& A \cdot \cot \theta_{1}=-\cot \theta_{1} \\
& A \cdot \cot \theta_{2}=-\cot \theta_{2}
\end{aligned}
$$

A stand for ratio between two impedance $A=\frac{Z_{2}}{Z_{1}}$ and $\theta$ represents the length of imepndance resonator. Total length of the filter, in form of cascaded structre, $\tau$ is given in equation 10.

$$
\tau=\frac{\theta_{2}}{\theta_{1}+\theta_{2}}
$$

Let $\theta_{t}=\theta_{1}+\theta_{2}$, and by substituing the equation 10 in equations 8 and 9 we get results as shown in (11) and (12).

$$
\begin{aligned}
& A \cdot \cot \left(\tau \theta_{t}\right)=-\cot \left(\theta_{t}(1-\tau)\right. \\
& A \cdot \cot \left(\tau \theta_{t}\right)=\tan \left(\theta_{t}(1-\tau)\right.
\end{aligned}
$$

From equations 11 and 12 we can conclude that results depend on $A$ and $\tau$, while $\theta_{t}$ can have multiple solutions. However, in design we always want to get optimized results. Ports in proposed design are symmetrical. Their design plays huge rule in the determining the size of the filter. Bending of the ports decreases the size of the filter, making it more compact for use in the microwave systems. However, one of the methods that can be used for analysis of the filter can be grouped in four phases. First phase is to develop third order lowpass filter, second phase is to transform lowpass filter into bandpass filter. Further, adjust bandpass filter to wanted frequency range. Finally, combine two bandpass filters into dual band pass filter to create one filter. Further, to increase performance of the filter defected ground structures (DGS) can be used. It is adjusted to the substrate integrated waveguide (SIW) resonator. Frequency ranges can be controlled by DGS, which is advantage compared to the previous controlling methods. Nevertheless, this type of the technology is found cheap and gives good performance response for many types of the filters. Using defected ground structure and substrate integrated waveguide results in higher $Q$ factor. However, losses mostly occur because of the finite conductivity of the bottom and top planes. 


\section{Conclusion}

Dual band pass with our proposed design is affordable, simple, small, compact device. This type of the filter plays huge rule in wireless communication and microwave technologies [15]. The main advantage of this filter is its small size with dual resonances. It is designed to work on two frequency bands at same time, simultaneously. Furthermore, this filter has good isolation between two operating bands. The response of the filter gives very stable results in first operating band while second is more sensitive and we have more variations. It has main applications in GSM (Global System for Mobile Communication) [16]. However, this design of the filter is not very attractive in GPS and FS systems, where almost definite cancellation of the noise is required. Multiband technology is very attractive to the next generations of technological development which includes multi-frequency devices. Simulations in Sonnet software are done in two minutes which represent the high-speed response. This feature is quite important for various applications. When designing and analyzing filters we must know that there is no perfect filter. There are many different influences from components of which filter is composed that affect output signal. However, we can design components in a way to get signal near ideal. They are usually composed of the lumped components (capacitors and inductors) and microstrip. Values for $\mathrm{S} 11$ parameter are $-20.70 \mathrm{~dB}$ and $-41.72 \mathrm{~dB}$ for $4.9 \mathrm{GHz}$ and $5.5 \mathrm{GHz}$ respectively. Values for $\mathrm{S} 12$ parameter are $-0.03 \mathrm{~dB}$ and $-2.91 \mathrm{e}-4 \mathrm{~dB}$ for $4.9 \mathrm{GHz}$ and $5.5 \mathrm{GHz}$, respectively. However, bandwidth can be optimized using different techniques. These filters have applications also in signal processing. From results we can obtain that this filter has its negative sides. However, they are very small and can be neglected. Filter with narrow band-pass are found demanding in modern technologies [17]. In modern wireless technology development of RF transceivers are very attractive for many scientists and researchers. They are capable to work on multiple frequencies.

\section{Acknowledgement}

We would like to thank to the International University of Sarajevo for providing us software accessibility.

\section{Reference}

[1] G. Sharma, S. Sharma, S. Bhullar, N. Kumar, S. Chauhan, "Design and simulation of compact hairpin band pass filter", International Journal of Modern Communication Technologies \& Research (IJMCTR), vol. 2, no. 4, 2014. ISSN:2321-0850

[2] D. Yadav, T. Moyra, K. Debbarma, "Microstrip Hairpin Bandpass Filter with Improved Out of Band Performance", Conference Paper, March 2014.

[3] Sonnet Suites, ver. 17.52. Syracuse, New York

[4] G. Zhao, F.-S. Zhang, Y. Song, Zi-Bin Weng and Y.-C. Jiao, "Compact ring monopole antenna with double meander lines for $2.4 / 5 \mathrm{GHz}$ dual-band operation", Progress in Electromagnetics Research, PIER, vol. 72, pp. 187-194, 2007.

[5] G.-L. Wu, W. Mu, X.-W. Dai and Y.-C. Jiao, "Design of Novel Dual-Band bandpass filter with microstrip meander-loop resonator and CSSR DGS", National Key Laboratory of Antennas and Microwave Technology Xidian University, Xi'an 710071, China

[6] D. M. Pozar, Microwave Engineering, United States of America: John Wiley \& Sons, Inc., 2012

[7] G. Zhang, J. Chen, J. Shi, H. Tang, H. Chu, and Z. Bao, "Design of Multilayer Balun Filter With Independently Controllable Dual Passbands," IEEE Microwave and Wireless Components Letters, vol. 25, no.1, pp. 10-12, 2015.

[8] H. Ma, Fundamentals of Electronic Circuit Design, 2005 
[9] L. ZHU, S. Sun and A. R. Li, Mirowave Bandpass filters for wideband communications, New Jersey: John Wiley \& Sons, Inc., 2012.

[10] G. Wu, L. Yang, and Q. Xu, "Miniaturized dual-band filter with high selectivity using split ring scheme," Electronics Letters, vol. 51, no.7, 570-572, April 2015.

[11] N. Ismail, T. S. Gunawan, S. Kartika, T. Praludi, E. A. Z. Hamidi, "Design of Microstrip Hairpin Bandpass Filter for $2.9 \mathrm{GHz}$ - 3.1 GHz S-band Radar With Defected Ground Structure," Malaysian Journal of Fundamental and Applied Sciences, vol. 14, 2018.

[12] Guan, X., Z. Ma, P. Cai, Y. Kobayashi, T. Anada, and G. Hagiwara, "Synthesis of dual-band filters using successive frequency transformations and circuit conversions," IEEE Microwave and Wireless Component Letters, vol. 16, no. 3, pp. 110-112, 2006.

[13] http://cpb.iphy.ac.cn/article/2015/cpb_24_10_107506/cpb150319f2_hr.jpg.html Last accessed on 8th January, 2019

[14] F. Xu, D. Z. Wang, M. Xiao, "A compact dual-band band pass filter with wide stop band using two resonators combined by via-hole”, Progress In Electromagnetics Research, January 2013.

[15] X. Xu, Y. Xuejun and a. L. Tao, "The design of 2.4GHz Microstrip Band Pass Filter", Advanced technology in Teaching, AISC, 2012.

[16] M. Rusdi, F. A. Batubara, R. Anugrahwaty, Junaidi, S. Alifuddin, B. Harianto, "Design of Dual-band Bandpass Filter for GSM $950 \mathrm{MHz}$ and GSM $1850 \mathrm{MHz}$ Applications using Lumped Component", Journal of Physics: Conference Series, 890 012049, 2017.

[17] N. N. Hussain, J. K. Ali, “A New Narrow Band Dual-Mode Miniaturized Bandpass Filter for Wireless Communication Systems”, Eng. \& Tech. Journal, vol.27, no.10, 2009. 